\title{
Tamsulosin bei Kindern nach ESWL wegen Nierensteinen
}

Die extrakorporale Stoßwellenlithotripsie (ESWL) führt bei Kindern mit Nierensteinen mittelfristig zu Steinfreiheitsraten von mehr als 90\%. Bei Erwachsenen wird in Ergänzung zur ESWL Tamsulosin eingesetzt, das als $\alpha$-Rezeptoren-Antagonist die glatte Muskulatur des Ureters entspannen und so die Austreibung der Steinfragmente verbessern soll. Ob das auch in einer pädiatrischen Population sinnvoll ist, haben Mediziner aus Ägypten untersucht. J Urol 2016; 195: 1284-1288

Tamsulosin trägt bei Kindern nicht zur besseren Ausscheidung von Nierensteinfragmenten nach ESWL bei. Zu diesem Ergebnis kommt die randomisierte Studie mit insgesamt 120 Kindern im medianen Alter von dreieinhalb Jahren (1-13 Jahre) mit medianer Steingröße von $1,2 \mathrm{~cm}(0,6-$ $2,2 \mathrm{~cm})$.

Einschlusskriterium war ein in der Bildgebung (Sonografie, Nativ-Röntgen, CT) gesicherter unilateraler singulärer Nierenbeckenstein. Jeweils 60 Kinder wurden einer von zwei Gruppen zugewiesen:

- nach der ESWL tägliche Gabe von Tamsulosin in einer Dosierung von $0,01 \mathrm{mg} / \mathrm{kg}$ über 3 Wochen (Gruppe 1) - keine Gabe von Tamsulosin (Gruppe 2)

Die ESWL erfolgte in Allgemeinanästhesie mit vergleichbaren technischen Einstellungen. Alle Kinder erhielten nach der Intervention Paracetamol $15 \mathrm{mg} / \mathrm{kg}$ alle 8 Stunden über 5 Tage. Beurteilt wurde die Steinfreiheit mittels Sonografie nach 3 und 6 Wochen, steinfrei war dabei definiert als Fehlen von Fragmenten $>3 \mathrm{~mm}$ Durchmesser und keine Erweiterung des Nierenbeckenkelchsystems. Bei nachweisbaren Fragmenten 6 Wochen nach der Erst-ESWL erfolgte eine zweite ESWLSitzung, nach weiteren 7-10 Wochen ggf. eine Ureteroskopie mit Extraktion der Steinreste.

Die Wissenschaftler fanden keine Unterschiede bei der Steinfreiheitsrate zwischen den beiden Gruppen. Die Rate be- trug insgesamt 82,5\% nach der ersten und $100 \%$ nach der zweiten Sitzung. Steinfragmente wurde nach der ersten ESWL bei 6 Kindern in Gruppe 1 und bei 8 Kindern in Gruppe 2 gefunden.Komplikationen traten bei $14,2 \%$ der Patienten auf und umfassten eine fieberhafte Pyelonephritis bei jeweils einem Kind pro Gruppe, die konservativ beherrschbar war, sowie Hämaturien über mehr als 1 Tag bei 9 Kindern der Gruppe 1 und 6 Kindern der Gruppe 2. Subkapsuläre oder perirenale Hämatome wurden nicht beobachtet. In der multivariaten Analyse errechneten sich als einzige unabhängige Prädiktoren für eine Steinfreiheit nach einer ESWL-Intervention die Größe der Steine und die Sichtbarkeit im Nativröntgenbild.

\section{Fazit}

Nach diesen Daten verbessert die zusätzliche Gabe von Tamsulosin bei Kindern nicht die Ausscheidung der Steinfragmente nach ESWL, fassen die Autoren zusammen. Möglicherweise liegt das auch an den bei Kindern elastischeren Ureteren, sodass die Steinaustreibung problemlos auch ohne medikamentöse Unterstützung gelingt. Kleinere Steine sprachen wie erwartet besser auf eine einmalige ESWL an, ebenso röntgendichte Steine. Zukünftige Studien sollten untersuchen, inwieweit die exakte chemische Zusammensetzung der Steine das Ansprechen auf die ESWL beeinflusst.

Dr. Elke Ruchalla, Bad Dürrheim 


\section{Kommentar}

\section{Tamsulosin bei Kindern nach ESWL ohne Effekt}

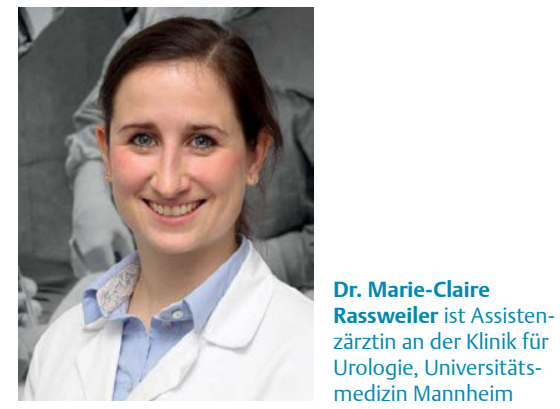

Der Einsatz von Tamsulosin bei Urolithiasis zur medikamentösen expulsiven Therapie wird in der Literatur aktuell viel diskutiert. Diese Standardtherapie bei Erwachsenen hat bei Kindern bisher eine geringe Evidenz, obwohl die Wirksamkeit der Alphablocker, insbesondere Tamsulosin, beschrieben ist [1-2]. Ein Review über den Einsatz von Tamsulosin bei Kindern zeigte einen positiven Effekt auf die Steinpassage bei Kindern und keine Nebenwirkungen durch die Medikamenteneinnahme [3].
In diesem Studiendesign wurden die Patienten nach strengen Selektionskriterien in 2 Gruppen a 60 Kindern randomisiert. Das Nachsorgeschema und die Dokumentation der Restkonkremente wurde u.a. durch low-dose Stein-CTs durchgeführt, um so eine genaue Evaluation möglicher Restfragmente zu bekommen. Hier wurde besonderen Wert auf die Steinfreiheit gelegt, welche bei Restfragmenten $<3 \mathrm{~mm}$ und keiner Dilatation des Hohlsystems ebenfalls sehr genau gehandhabt wurde.

Als Schwäche der Studie kann die fehlende Steinanalyse der behandelten Konkremente genannt werden. Hier wäre interessant gewesen, ob ein Zusammenhang zwischen der zweiten notwendigen ESWL und der Steinzusammensetzung dargestellt werden könnte. Als weitere Limitation erwähnt der Autor eine fehlende dritte placebokontrollierte Gruppe zur Komplettierung der Studie.

Shahat et al. zeigt klar, dass bei Kindern nach ESWL eine Therapie mit Tamsulosin keinen Vorteil gegenüber der alleinigen Gabe von Paracetamol bzgl. der Steinpassage gibt. Auch wenn ältere Studien gegenteilige Ergebnisse zeigten, besteht insgesamt keine eindeutige Evidenz über den
Nutzen der Alphablocker-Therapie bei Kindern.

\section{Fazit}

Bei Kindern nach Stoßwellentherapie bei Nephrolithiasis ist eine Therapie mit Tamsulosin nicht sinnvoll. Bei komplexen Fällen kann diese dennoch hilfreich sein und sicher eingesetzt werden.

\section{Dr. Marie-Claire Rassweiler, Mannheim}

\section{Literatur}

1 Aydogdu O, Burgu B, Gucuk A et al. Effectiveness of doxazosin in treatment of distal ureteral stones in children. J Urol 2009; 182 : 2880-2884

2 Mokhless I, Zahran AR, Youssif M et al. Tamsulosin for the management of distal ureteral stones in children: a prospective randomized study. J Pediatr Urol 2012; 8: 544-548

3 Velázquez N, Zapata D, Wang HH et al. Medical expulsive therapy for pediatric urolithiasis: Systematic review and meta-analysis. J Pediatric Urol 2015; 11: 321-327 